\title{
Thoracic neuroblastoma presenting as a cystic hygroma
}

\author{
D J GANICK, ${ }^{*}$ M B KODROFF, $\dagger$ H G MARROW, $\ddagger$ C T HOLBROOK,${ }^{*}$ AND W J PORIES $\S$
}

Departments of *Pediatrics, §Surgery, $\nmid$ Radiology, and $\ddagger$ Clinical Pathology, East Carolina University School of Medicine, Greenville, North Carolina, USA

SUMMARY A case of thoracic neuroblastoma with extension into the supraclavicular fossa is described. By physical examination and ultrasound the patient was thought to have a cystic hygroma with mediastinal extension. A 'cystic' neck mass may be the initial presentation of a mediastinal neuroblastoma.

Neuroblastoma is the third most common tumour of childhood. ${ }^{1}$ The initial clinical presentation of the tumour is remarkable for its protean manifestations. In addition to the frequent finding of a firm mass on physical examination of the child, the initial presentation also may be characterised by symptoms and findings related to metastatic spread of the tumour or its metabolic effects.

We report a case of an infant with mild respiratory distress and a neck mass that felt like a cyst located in the supraclavicular area. The ultrasound findings supported the diagnosis of cystic hygroma. On pathological examination it was found that the presumed cystic mass was a primitive neuroblastoma with haemorrhage and necrosis. There have been previous reports of abdominal neuroblastoma diagnosed preoperatively as cystic masses. ${ }^{2}{ }^{3}$ A cystic neck mass therefore can be added to the list of presentations of thoracic neuroblastoma. ${ }^{4}$

\section{Case report}

A 6 month old boy was admitted to hospital with fever and congestion. A chest radiograph showed right upper lobe consolidation, possibly a mass. On physical examination he had right supraclavicular adenopathy. The patient was transferred to the Children's Hospital of Eastern North Carolina because of the possibility of a chest tumour.

The patient was examined and found to have several small $(1-2 \mathrm{~cm})$ firm, nodular like masses as well as several small cystic masses in the right supraclavicular area. The total mass was about $3 \times 3 \mathrm{~cm}$ and transilluminated. The rest of the physical examination was normal. A repeat radiograph showed the previously described extrapulmonary mass with deviation of the trachea to the left, indicating mass effect in the mediastinum (fig 1). Ultrasound of the neck showed a supra- clavicular mass with mediastinal extension consisting of multiple separate fluid filled spaces with. echogenic material within some of the cysts (fig 2). The findings were compatible with a cystic hygroma, or, less likely a cystic teratoma.

The patient was admitted three weeks later for excision of the cystic hygroma. At surgery multiple cystic like nodules were removed from the right supraclavicular fossa. Grossly the tumour nodules were lobulated and contained grey, firm, homogeneous areas on cut section. Extensive areas were haemorrhagic and necrotic. Microscopically the neoplasm was highly cellular and consisted of cells slightly larger than mature lymphocytes. Cells were present in sheets and often clustered about a central area of eosinophilic, filamentous material (Homer Wright rosettes). This filamentous material and the individual cells stained strongly with neurofilament and neuron specific enolase. Mitoses were frequent. Eléctron microscopic examination was remarkable for glycogen and numerous electron dense neurosecretory type granules, features characteristic of neuroblastoma.

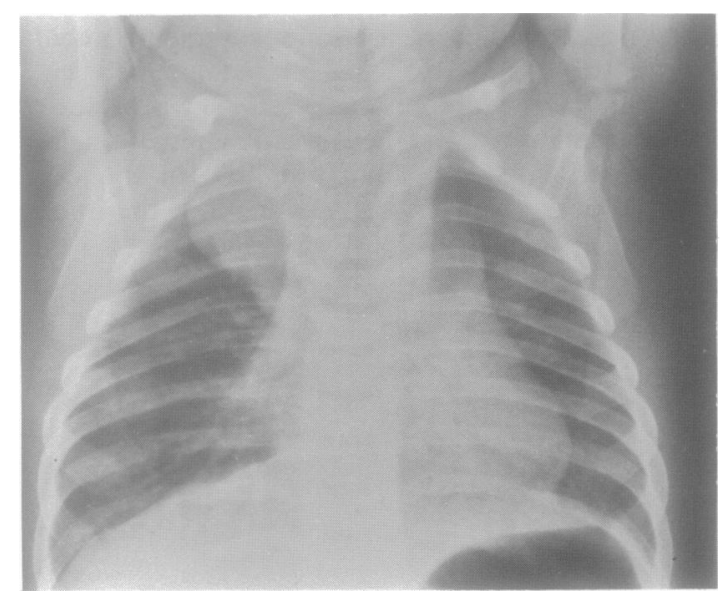

Fig 1 A frontal radiograph of the chest showing a soft tissue mass displacing the trachea both within the neck and within the thorax which on a lateral radiograph (not included) was displacing the trachea slightly anteriorly. No calcification is obvious on the plain films. 


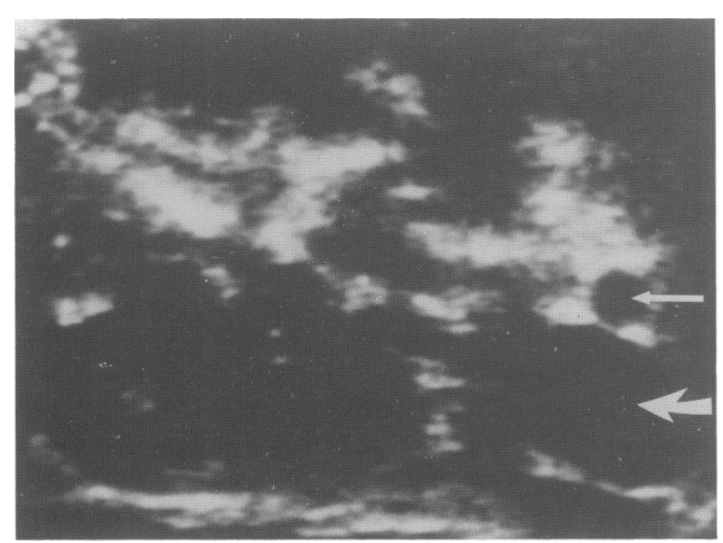

Fig 2 A transverse ultrasound study of the neck showing a mass consisting of multiple fluid filled spaces as evidenced by the lack of internal echoes, strong backwall, and increased through transmission of sound. There are solid elements within some of the fluid filled spaces. Thick arrow indicates jugular vein; thin arrow indicates carotid artery.

One week later the patient underwent a thoracotomy to debulk the remaining intrathoracic tumour. At surgery a large tumour posterior to the oesophagus was dissected from the superior vena cava, inominate artery and vein, as well as carotid and subclavian arteries. The tumour was found to extend into the vertebral foramina $\mathrm{T} 4, \mathrm{~T} 5, \mathrm{~T} 6$, and $\mathrm{T} 8$ and small remnants were left. The pathology of the tumour was similar to the previous biopsy specimen and showed a neuroblastoma with rare ganglionic differentiation.

Preoperatively the urinary vanillylmandelic acid concentration was $103 \mu \mathrm{g} / \mathrm{mg}$ creatinine (upper limit of normal, $15 \mu \mathrm{g}$ ) and the homovanillic acid concentration was $8 \mu \mathrm{g} / \mathrm{mg}$ creatinine (upper limit of normal, $37 \mu \mathrm{g}$ ). The postoperative metastatic workup, which included a bone marrow biopsy, ultrasound of the abdomen, a skeletal survey and bone scan, gave negative results for tumour.

The patient received five courses of chemotherapy given every three weeks; this consisted of daily oral cyclophosphamide $\left(150 \mathrm{mg} / \mathrm{m}^{2}\right)$ for seven days followed on day eight by adriamycin $\left(35 \mathrm{mg} / \mathrm{m}^{2}\right)$. Complete re-evaluation after five courses of treatment showed that the patient had no evidence of tumour. He has had no recurrence of the tumour in the 18 months since diagnosis.

\section{Discussion}

Our case is interesting from several aspects of differential diagnosis. Because of the location of the cystic like mass in the supraclavicular area, the patient was thought to have a cystic hygroma. ${ }^{5}$ Thoracic extension of the neck mass occurs in $10 \%$ of the cases. ${ }^{6}$ The sonographic findings in our case appeared to confirm the cystic nature of the mass. The echogenic areas within the mass were compatible with lymphangiomatous malformations. Another diagnostic consideration was a cervical teratoma, which can cause respiratory distress by tracheal compression.

There have been reports of cystic abdominal masses diagnosed by ultrasound, which at surgery were found to be neuroblastomas. In these cases the cystic masses were thought preoperatively to represent cystic changes in the adrenal gland resulting from haemorrhage. ${ }^{23}$ Biopsy specimens taken from the cases reported have shown, like those from our patient, haemorrhage, necrosis, and a variable amount of microcystic transformation within the neuroblastoma.

The clinical presentation of neuroblastoma is remarkable for its variety. ${ }^{4}$ Patients with thoracic neuroblastoma may present with severe respiratory distress due to tracheal compression from the tumour. Neurologic dysfunction due to extension of the tumour into the vertebral canal and Horner's syndrome due to involvement of the cervical ganglia are other clinical presentations in the thorax. ${ }^{4}$ Occasionally a patient is found to have a thoracic neuroblastoma on a routine chest radiograph. Our case adds another clinical presentation for thoracic neuroblastoma, namely a cystic like neck mass. When encountering children with cystic neck masses containing nodular (presumed lymphatic) tissue components and notable extension into the mediastinum one should consider neuroblastoma.

\section{References}

1 Young JL, Miller RW. Incidence of malignant tumors in US children. J Pediatr 1975;86:254-8.

2 Atkinson GO, Zaatari GS, Lorenzo RL, et al. Cystic neuroblastoma in infants. Radiographic and pathologic features. $A J R$ 1986;146:113-7.

${ }^{3}$ Hendry GMA. Cystic neuroblastoma of the adrenal glanda potential source of error in ultrasonic diagnosis. Pediatr Radiol 1982;12:204-6.

${ }^{4}$ McLatchie GR, Young DG. Presenting features of thoracic neuroblastomas. Arch Dis Child 1980;55:958-62.

${ }^{5}$ Pounds LA. Neck masses of congenital origin. Pediatr Clin North Am 1981;28:841-4.

' Pokorny WJ. Mediastinal masses in infants and children. J Thoracic Cardiovasc Surg 1974;6:869-75.

Correspondence to Dr DJ Ganick, Department of Pediatrics, East Carolina University School of Medicine, Greenville, North Carolina 27858-4354, USA.

Accepted 21 March 1988 